\title{
TWIICE One powered exoskeleton: Effect of design improvements on usability in daily-life as measured by Cybathlon race performance
}

Tristan Vouga ( $\nabla$ tristan.vouga@twiice.ch )

TWIICE SA https://orcid.org/0000-0001-7244-133X

Jemina Fasola

TWIICE SA

Romain Baud

SONCEBOZ SA

Ali Reza Manzoori

EPFL: Ecole Polytechnique Federale de Lausanne

Julien Pache

TWIICE SA

Mohamed Bouri

EPFL: Ecole Polytechnique Federale de Lausanne

\section{Research}

Keywords: Powered exoskeleton, wearable robotics, powered gait orthosis, spinal cord injury, overground walking, gait, exoskeleton training

Posted Date: November 1st, 2021

DOl: https://doi.org/10.21203/rs.3.rs-995192/v1

License: (9) This work is licensed under a Creative Commons Attribution 4.0 International License.

Read Full License 


\section{Abstract \\ Background}

Spinal cord injury leading to paraplegia affects the mobility and physiological well-being of nearly one in a thousand people. Powered exoskeletons can temporarily restore the ability to walk. Their relevance in daily life is still limited because of low performance beyond even ground. Cybathlon is an international competition promoting improvements in assistive technology. In this article we present the latest design and results of testing of TWIICE One 2018, one of the competing devices in the 2020 race.

\section{Methods}

A person with a motor-complete spinal cord injury at thoracic level T10 participated as race pilot. Training ahead of the race took place over one week at a rate of 2-5 hours per week. Time to perform each of the 7 tasks of the competition was recorded together with the number of repetitions. Performance is compared over training time and against 2016 race results.

\section{Results}

Progression was observed in all tasks and accounted for by both user training and technology improvements. Final competition rank was second out of 7 participating teams, with a record time of 4'40" min. This represents an average of $40 \%$ improvement with respect to comparable obstacles of the 2016 race, explaining the two ranks of improvement since then.

\section{Conclusion}

These results help understand which features had a positive impact on real life performance of the device. Understanding how design affects performance is a key information to create devices that really improve the life of people living with paraplegia.

\section{Background}

Restoring functional mobility in people with motor-complete spinal cord injury (SCl) is one of the feasible applications of powered exoskeletons [1], [2] in which significant progress has been made over the past decade. Serious efforts for the development of such devices date back at least to the late 20th century [3], and advances in robotics-related technologies have facilitated these developments. Significant improvements have been achieved since the early proof-of-concept studies showing the feasibility of powered orthoses to enable SCI patients' ambulation [4], and several commercialized or market-ready solutions already exist today [5]-[11]. Studies have also clearly shown the desirability of these devices for people with SCI [12], [13]. 
These devices can be used by a wide range of subjects [14] and offer numerous advantages compared to passive devices and wheelchairs, including improved mobility [15], enabling locomotion over different types of terrain [16], and increased motivation for physical activity [13], [17], which in turn lead to physical [18], [19], emotional/mental and social benefits [20]-[22]. Improved mobility, social functionality and the ability to carry out activities of daily living (ADLs) are important factors for improved quality of life in people with $\mathrm{SCl}$ [23], [24]. Various other potential benefits and advantages of using such devices have also been widely reported in the literature [25].

However, despite all of the positive points, these devices are not yet recognized as suitable for completely independent everyday use rather than use in clinical and controlled settings [26]-[28], despite preliminary evidence for the feasibility of their use in realistic settings [25], [29]. Factors such as safety concerns, the need for supervision and/or external supporting structures [26], reliance on crutches, slow or robot-like walking [12], high prices [30] and their limited availability [27] render the widespread adoption of such devices for everyday use impractical. Moreover, the risk management and regulatory systems for these devices are not mature yet and require better understanding of the various aspects related to their use [31]. These issues highlight the necessity of further development of the exoskeletons [26] and more studies on their usage in realistic settings [31]. Improvements in controllers and trajectories can also further improve the functional outcomes [32].

One of the major drivers for the development and adaptation of assistive technologies to real-world scenarios in the recent years has been the CYBATHLON, a competition for people with physical disabilities using advanced assistive technologies [33]. Other than promoting the development of assistive technologies, CYBATHLON has raised public awareness and generated public interest in this field [34] by showcasing the devices and their use. Also, it has encouraged scientific exchange [34] and has provided some standardized metrics for comparison and benchmarking of the technologies and devices in terms of performance [35], [36]. One of its disciplines is the powered exoskeletons race [37] which consists in a set of tasks representative of the ADLs. The tasks of this race have guided the development of several lower-limb exoskeletons, as previously reported in the literature [38]-[40]. One of these exoskeletons is TWIICE-2018, which empowered the silver medalist in CYBATHLON 2020.

The development of TWIICE started in 2015, with the aim of creating a lower limb exoskeleton for paraplegic people with a modular, compact and lightweight structure. With the principle of simplicity in mind, only the hip and knee joints were selected to be actuated. The ankle has been locked. The lack of this degree of freedom (DOF) was compensated with a curved sole, one of the unique features of TWIICE. The first version of the device in 2016 [41], has been driven by our participation in the first CYBATHLON the same year. The evolution of TWIICE on the level of software, hardware and electronics kept progressing in close interaction with the pilot, with regular tests and participations in the intermediate CYBATHLON Experience events. These incremental efforts of research and development culminated in TWIICE-2018, which is described in this paper.

\section{Methods}




\subsection{Mechanical design}

\subsubsection{Overview}

TWIICE is a powered hip-knee-ankle-foot orthosis comprising four actuated joints in total and no passive joint. The structure maintains the user's lower-limb from torso to foot, using five attachment points: a thoracic belt, a waist belt, thigh and tibial cuffs and foot straps (Figure 1). The active joints mobilize the hips and knees in the sagittal plane, so that gait motion is made possible to users with a motor-complete spinal cord injury. Batteries as well as controls and power electronics are located in the back of the user in a robust enclosure rigidly mounted on the device's back structure. Adjustment possibilities include length of the tibial and thigh segments, width of the pelvic structure and ab-/adduction angle. Several height positions of the thoracic belt are also possible to adapt to the user level of injury. Power and signal distribution to the actuators is made through multipolar cables protected with mechanical and electromagnetic shielding. Power and digital lines are combined in the same cable assembly using Fischer Connectors' Alulite connectors [42]. Peripherals include forearm crutches with user input device and an off-the-shelf smartwatch. Construction materials are primarily long carbon fiber reinforced epoxy built in sandwich arrangement for the thigh and tibia segments and aluminum for the pelvic structure. User attachment points are made of technical breathable fabric and lightweight foam. In total, the device weighs $16 \mathrm{~kg}$ including batteries.

\subsubsection{Design philosophy}

The architecture and design philosophy of TWIICE is revolving around simplicity, with the underlying intention to achieve more robustness and lighter weight. From the number of actuators to the manufacturing methods, a minimalistic approach was adopted in design decisions. This strategy led to lower implementation costs, reliable operation over the years and light weight. Over 20'000 steps were taken with the prototype during development tests and no hardware failure occurred. Hardware maintenance remained low with no significant part replacement apart from foot sole and one fatigue failure of the trigger used for user input.

\subsubsection{Actuation}

The four actuators are identical in design. They consist of a brushless DC motor and a cycloidal gear with ratio 1:51 custom made by the company Sonceboz SA [43]. The design rated torque is $100 \mathrm{Nm}$, limited by the mechanical capacity of the gear. The motor's custom design had the following key design objectives: high torque density, low backdriving torque, safe mechanical failure mode. The resulting key design performance metrics are detailed in Table 1. Other features include: a relative position encoder on the motor's rotor, an absolute position encoder measuring the joint angle, signal conditioning and sampling performed locally with digital communication bus to main board, mechanical stops at anthropomorphic joint limits in flexion and extension. The cycloidal gear has a blocking failure mode in case of overstress, 
offering an inherently safe design. The total weight per actuator is $1.63 \mathrm{~kg}$, with an external diameter of $85 \mathrm{~mm}$ and axial length of $55 \mathrm{~mm}$.

\subsubsection{Feet}

Simplicity and light weight were, among other, achieved through reduction of the number of degrees of freedom. To make up for the lack of mobility at the ankle, a special sole shape was designed to allow for easy lateral weight shift and rolling on the floor during stance phase. In order to manage rough terrain and navigating narrow spaces, the foot design was further improved with respect to TWIICE One 2016. Compliance was added in the sole to allow the fore part of the foot to flex with respect to the main base at the metatarsal level. The overall length and height of the sole were also reduced to facilitate interaction with the ground and irregularities.

Table 1

Technical specifications, typical preparation time, walking speed and adjustability of the exoskeleton TWIICE ONE 2018

\begin{tabular}{|lll|}
\hline Specifications & \multicolumn{2}{l|}{ Unit } \\
\hline Weight & $\mathrm{kg}$ & 16 \\
\hline Intermittent peak torque & $\mathrm{Nm}$ & 100 \\
\hline Max. joint velocity & $\mathrm{rpm}$ & 40 \\
\hline Battery life & $\mathrm{h}$ & 3 \\
\hline Typical preparation time & $\mathrm{s}$ & $60 \mathrm{~s}$ \\
\hline Typical don/doff time & $\mathrm{s}$ & $120 / 75$ \\
\hline Typical walking speed & $\mathrm{m} / \mathrm{s}$ & 0.32 \\
\hline Hip width & $\mathrm{m}$ & 0.340 to 0.400 \\
\hline Thigh length & $\mathrm{m}$ & 0.385 to 0.455 \\
\hline Shank length + ankle height & $\mathrm{m}$ & 0.431 to 0.533 \\
\hline
\end{tabular}

\subsection{CONTROL}

\subsubsection{User interface}

TWIICE is controlled by the pilot via two main input devices: (i) buttons on the crutch handle and (ii) an Android smartphone or smartwatch installed with an interface application (Figure 1, top inlay). The set of buttons on the crutch include 3 normal push buttons and a trigger button. They are pressed respectively with the thumb and the index finger, while the hand holds the crutch handle. The push buttons are mostly used to navigate between the modes and exit them, while the trigger is used to initiate actions (e.g. entering a mode or taking a step). The smartphone/smartwatch is used to send higher-level commands 
that are not used during walking, such as (de)activating the motors or shutting down the exoskeleton. Besides this functionality, it principally serves as a visual feedback device, displaying the selected/active mode to the user, and the battery state. In order to mitigate the risk of connection loss between the smartphone/smartwatch and the exoskeleton, in the 2018 version, a wired LCD display was integrated into the crutch. The name of the currently selected/active mode is displayed on this extra screen, which is wired to the embedded computer of the exoskeleton.

\subsubsection{Controller behavior}

As described in [41], the control of TWIICE is based on several pre-recorded trajectories, selected with specific operation modes. When no mode is selected, the exoskeleton stands still. In this state, the mode can be selected using the "up" and "down" buttons of the crutches, and activated using the trigger. When a mode is active, the trigger can be pressed to initiate the main action (making a step, standing-up, etc.). Pressing the "exit" button ends the current mode. The legs go back to the standing position with a movement defined by the current mode.

Since TWIICE One 2018, depending on the currently selected operation mode, the "up" and "down" buttons can trigger a mode-specific secondary action. This feature is used by the following new modes:

- Periodic gait mode: "up" and "down" switch smoothly from a gait trajectory variant to another (e.g. different steps size, stairs height). This avoids the need for numerous gait modes.

- Tilted path mode: "up" and "down" offset vertically the foot locus of one foot with respect to the other one. This is useful to walk through a terrain inclined laterally, such as the tilted path obstacle of the CYBATHLON.

- Rough terrain mode: "up" triggers a higher and longer step to walk over a small obstacle. "Down" triggers a higher step to step on a small obstacle. These special steps have been designed specifically for the "rough terrain" obstacle of the CYBATHLON 2020.

The main motivation for the pre-defined trajectories is that they are predictable and can be learnt by the user. This facilitates the contribution of the upper limbs to the gait. However, there is no automatic adaptation to the terrain. Increasing the diversity of the gait trajectories addresses more situations but results in a longer time to switch from one mode to another, and is also more error-prone. For the CYBATHLON 2020, 9 modes were available to the TWIICE pilot to overcome the six obstacles (sofa sitting, normal gait, fast gait, rough terrain, stairs ascent, stairs descent, tilted path, slope ascent, slope descent).

\subsubsection{Gait trajectories design}

As previously explained, the architecture of the controller is such that the trajectories can be modified independently of the controller itself and be provided as inputs to it, at runtime. This allows quick and easy testing of different trajectories without any significant downtime in between, which is very important for iterative adaptation of the trajectories for different tasks. Such iterations sometimes were even made several times in the course of a single training session. Therefore, being able to generate/modify and test trajectories in a "rapid prototyping" manner is a key feature. 
For TWIICE One 2016, the design of the gait trajectories was done from a list of joint angles associated to the strides in the gait cycle. This input was given to a MATLAB script (Mathworks Inc., Natick, MA, USA) that interpolates them over the full gait cycle with a piecewise cubic spline, and displays the corresponding joint angles, joint velocities and the resulting foot locus obtained by forward kinematics. It eventually generates $\mathrm{C}$-code with the spline coefficients, to be integrated in the TWIICE controller source code, that should be recompiled. The input points are tuned manually until the foot locus, joints angles and velocities are deemed satisfactory. The whole process is time-consuming, typically needing 15 minutes for a small change in the gait trajectory.

Therefore, to further streamline this procedure, a dedicated application was developed with an interactive graphical user interface that allows designing and modifying the foot locus trajectory, while displaying the joint-level outcomes in real-time. The reachability map of the exoskeleton is also displayed to prevent designing trajectories that require exceeding joint angle limits. The joint velocity over time is also given to check that the maximum speed of the actuator is not overcome. The application then outputs foot locus trajectory files that can be directly used by the exoskeleton's high-level control software, and inverse kinematics are solved on-board the exoskeleton.

Figure 2 shows the joint kinematics of the hip and knee, as well as the torques for walking, stairs and slope ascent. One main difference for the walking pattern is that the stance phase starts with the knee slightly bent to give some forward momentum when the foot hits the ground. The maximum torque required for ambulating is $50 \mathrm{Nm}$. The stairs ascent is done two at a time and a peak torque of $95.5 \mathrm{Nm}$ is reached in the stance phase during the knee extension. As in 2016, the stairs descent uses the inverse trajectories of the stair ascent and the pilot goes down backward due to the ankle's limitations and for safety reasons. The ramp ascent mostly solicits the hip with a maximum torque of $79.2 \mathrm{Nm}$ at the end of the stance phase.

\section{Results}

\subsection{Pilot}

One T10 sensorimotor complete spinal cord injured (AIS A) pilot took part to the development, test, and validation of the usability of the exoskeleton. The test pilot was the same athlete as in the CYBATHLON 2016 and has therefore regularly trained (i.e. sit-to-stand transition, in- and outdoor walking and stairs climbing) with the exoskeleton for four years. She has sufficient voluntary control of shoulders, arms and neck to keep her upper body upright and use the crutches to balance, but needs assistance, provided by an upper belt, to maintain her trunk upright. The pilot does not have any restriction in the range of motion of the hips and knees, but has some ankle stiffness that decreases progressively when loaded regularly. Detailed information of the pilot is found in Table 2.

The testing and development was performed in agreement with the guidelines for technical assistance devices/medical devices from Swissethic. The test users gave informed consent to participate in the tests 
and the training.

Table 2

Detailed information on the test pilot

\begin{tabular}{|lll|}
\hline Specifications & Unit & Pilot \\
\hline Age & Years & 48 \\
\hline Weight & $\mathrm{kg}$ & 45 \\
\hline Height & $\mathrm{m}$ & 1.58 \\
\hline Gender & & Female \\
\hline Years post injury & Years & 13 \\
\hline Level of injury & & T10-T11 \\
\hline AlS classification & & A \\
\hline Self-reported clinical syndrome & & Neuropathic pain, low spasms \\
\hline $\begin{array}{l}\text { Previous experiences with } \\
\text { exoskeleton }\end{array}$ & TWIICE One 2016- & CYBATHLON 2016 \\
& 2018 & CYBATHLON experience 2017, 2018, \\
\hline
\end{tabular}

\subsection{CYBATHLON 2020}

Since the pilot is experienced and had very limited availability ahead of the competition, only five intensive training sessions of about two hours each were planned a week prior to the CYBATHLON 2020 competition. The obstacles were mostly trained in sequence and in the same order as in the competition to account for the exertion factor along the race progression (Figure 3). The race comprised 1) "sit \& stand" task asking the pilot to sit down and get up from a sofa of a height of $460 \mathrm{~mm}$ and walk to a table to stack cups, 2) "slalom" task challenging the pilot to navigate around pieces of furniture, 3) "rough terrain" task which required to accurately control the feet positioning, 4) "stairs" task testing the ability of the exoskeleton to ascend and descend stairs, 5) "tilted path" task including perpendicular tilted ground to the walking direction, and 6) "ramp \& door" asking the pilot to ascend a $20^{\circ}$ slope and descend a $15^{\circ}$ degree slope and navigate through a door while walking on a flat ground. The best times of similar CYBATHLON 2016 obstacles, training sessions as well as the three CYBATHLON 2020 rounds are shown in Figure 3. We can observe that the "sit \& stand" task was up to $37 \mathrm{~s}$ faster than in 2016 although an additional standing agility task was added. The navigation task called "slalom" increased in difficulty with bulkier objects to circumvent and narrower spaces. However, time needed to clear this task decreased by about $20 \mathrm{~s}$ between 2016 and the first training session. To increase gait stability and preserve the pilot from fatigue, it was decided to reduce the pace after the first training. The "rough terrain", not performed in 2016 , was cleared with a best time of $36 \mathrm{~s}$. This time was drastically reduced during session 3 thanks to foot design modification and a new stepping strategy. The foot sole being shorter with an additional elastic passive joint at the toes, longer steps with a high foot clearance could 
be taken over the obstacles. Moreover, the tip of the foot could bite into the unevenness of the ground. The "rough terrain" was the most trained task with the "ramp and door" task. The "stairs" task was exactly the same as in 2016, and the time record was beaten by 55 s. Having no hip abduction/adduction nor pronation/supination in the ankle, the "tilted path" could be cleared in $42 \mathrm{~s}$ thanks to an asymmetrical gait pattern. The pilot could select with the crutches button how much the upstream leg had to remain more bent during the entire gait cycle. Finally, the "ramp \& door" task was passed $58 \mathrm{~s}$ faster than in 2016. The flexible tip of the foot sole allowed a better posture and a better grip on the ground avoiding the whole body to rotate when in single stance. The record time of $4{ }^{\prime} 40^{\prime \prime}$ for the whole race was during the second round of the competition. This represents an average of $40 \%$ improvement with respect to comparable obstacles of the 2016 race.

\subsection{General metrics}

The 10-meter walk test (10MWT) was used to measure the typical and fastest walking speed. The test pilot walks at $0.32 \mathrm{~m} / \mathrm{s}$ with the normal gai mode, which has a shorter step length than the fast gait allowing to negotiate turns with ease. This mode is mostly used in narrow spaces or in a crowded environment. Fast gait mode is used for walking in a straight line. The pilot reaches a maximum speed of $0.40 \mathrm{~m} / \mathrm{s}$ which is $0.04 \mathrm{~m} / \mathrm{s}$ faster than in 2016 and above the mean for four studies with other devices including a total of 53 AIS A patients (mean $=0.35 \mathrm{~ms}-1, \mathrm{SD}=0.12$ ) [16], [20], [44], [45]. At such speed, the gait cadence is $1.1 \mathrm{step} / \mathrm{s}$.

\section{Discussion}

TWIICE-2018 is an evolution of TWIICE-2016 and keeps its most important design points, that are the composite mechanical structure and the 4 actuated DoFs. In addition, the rolling sole of the foot compensates for the lack of a flexible ankle, and allows turning easily, thanks to the small area of contact with the floor. The control strategy also remains position-control, with operation modes to set the joints trajectory. Every step or other action is triggered by the fingers of the pilot, by the 4 buttons on the right crutch handle.

This simple design makes TWIICE lightweight and predictable. However, due to the lack of actuation at the ankle and the pre-defined trajectories, walking across unstructured terrain or an obstacle not handled by a specific control mode is difficult for the pilot. The contribution from the arms and the crutches is then increased, to ensure the stability.

TWIICE-2018 brings an improved mechanical design to increase the joints torque and the compactness. This was possible thanks to the custom integrated actuator, including the motor, gears and sensory electronics. Compliance was also added to the rolling feet.

The embedded controller was also extended with additional operating modes to address two new obstacles of the CYBATHLON: the "tilted path" (that was different in 2016) and the "rough terrain". The gait trajectories were optimized by trial-and-error thanks to a desktop application allowing quick 
iterations. Manual control of the exoskeleton by the pilot using the buttons on the crutch also turned out to be a good strategy and was maintained in the new version. This method of control, in combination with predefined trajectories, has rendered the interaction between the pilot and the exoskeleton fairly simple, thanks to the predictability of its behavior. This, however, comes at the cost of inflexibility in the face of uncertainties in the environment. This problem could be mitigated by providing several locomotion modes, each with a trajectory designed for a specific type of terrain.

The main outcome is a better time to go through the CYBATHLON obstacles, compared to the 2016 edition.

It can be noted that the gait speed did not increase significantly. This is due to the gait trajectories being triggered step by step requiring a pause in the middle of the stride. Indeed, all the joints are fully stopped between each step, so time is lost to decelerate, wait for the user input, and accelerate again.

The main limitation of these results is that the performance of TWIICE was assessed with a single pilot only. Chosen for her athletic condition, her performance is not necessarily representative of most people affected by paraplegia. The performance indices exposed here should then be considered as the maximum achievable, not the typical one.

In the future, the controller of TWIICE will be improved to support a continuous gait, to increase the ambulation velocity. To allow walking in unstructured terrain, adding more gait trajectories is not sustainable because of the increased time to select them, and the increased risk of error. Terrainawareness and dynamically-computed gait could be implemented if extra sensing is added to TWIICE, possibly using a LIDAR distance sensor.

\section{Conclusions}

In this paper, we presented the evolution of TWIICE One, a powered hip-knee-ankle-foot orthosis for persons with $\mathrm{SCl}$, and reported the achieved functional outcomes in terms of performance in the CYBATHLON competition. The outcome of 2 years of development, TWIICE One 2018 is actuated at the hip and knee levels and benefits from a curved sole similar to its 2016 predecessor. However, the design has been improved with structural flexibility of the feet, adjustability of segment lengths and more powerful actuators. The minimalistic design approach proved to be a successful trade-off, resulting in a low-cost implementation, compactness, robustness against failures and low maintenance requirements thanks to fewer components.

In addition to the mechanical modification, some of the locomotion modes have been improved with parameters that are tunable by the pilot during operation. The accelerated optimization of the predefined trajectories, thanks to an efficient design interface, was found to have remarkable potential in improving the overall speed and ease of use of the exoskeleton. 
The outcome of these improvements was reflected in a notably faster performance in CYBATHLON 2020 and winning the second place, with a reduction of up to $48 \%$ in the time required for some of the tasks compared to 2016. Despite the modifications in hardware and software, our pilot was not only able to easily adapt to the changes, but also improved her performance significantly with minimal training. This was possible thanks in part to the pilot's previous experience with the exoskeleton, even after a long hiatus imposed by the sanitary restrictions due to the COVID-19 pandemic. This highlights the importance of initial training for exoskeleton users, and suggests that the acquired skills can persist over long periods of time.

\section{Declarations}

\section{Ethics approval and consent to participate}

Ethical review and approval was not required according to the guidelines of in agreement with the guidelines for technical assistance devices/medical devices from Swissethic.

\section{Consent for publication}

The individual who participated in this study provided written consent to publish identifiable details in this article under the CC BY 4.0 license.

\section{Availability of data and materials}

All data is presented in the article.

\section{Competing interests}

TV, JP and JF are employees of TWIICE SA, a company commercializing products derived from the present work. TV, JF, RB and MB are authors of patents covering aspects described in this article.

\section{Funding}

Funding for this research came from Sonceboz SA, the Swiss National Center for Competence in Research Robotics Spin Fund, the EPFL Innogrant programme, Fischer Connectors SA, and Bâloise Group.

\section{Authors' contributions}

TV, JF, RB and AM equally contributed to the drafting of the manuscript. TV and JP predominantly conceived the mechanical design of the exoskeleton. RB designed the controllers. JF analyzed and 
interpreted the collected data. MB helped in drafting the manuscript and critically revising it. All authors contributed to the article and approved the submitted version.

\section{Acknowledgements}

The authors wish to thank the test-pilot who volunteered to part in the experiment.

\section{Abbreviations}

$\mathrm{SCl}$ Spinal cord injury

ADL Activities of daily living

DOFs Degrees of freedom

\section{References}

1. E. Hong et al., "Mobility Skills With Exoskeletal-Assisted Walking in Persons With SCl: Results From a Three Center Randomized Clinical Trial," Front. Robot. Al, vol. 7, p. 93, 2020, doi: 10.3389/frobt.2020.00093.

2. R. B. van Dijsseldonk, H. Rijken, I. J. W. van Nes, H. van de Meent, and N. L. W. Keijsers, "Predictors of exoskeleton motor learning in spinal cord injured patients," Disabil. Rehabil., vol. 43, no. 14, pp. 1982-1988, Jul. 2021, doi: 10.1080/09638288.2019.1689578.

3. M. Vukobratovic, D. Hristic, and Z. Stojiljkovic, "Development of active anthropomorphic exoskeletons," Med. Biol. Eng., vol. 12, no. 1, pp. 66-80, Jan. 1974, doi: 10.1007/BF02629836.

4. Y. Ohta, H. Yano, R. Suzuki, M. Yoshida, N. Kawashima, and K. Nakazawa, "A two-degree-of-freedom motor-powered gait orthosis for spinal cord injury patients," Proc. Inst. Mech. Eng. [H], vol. 221, no. 6, pp. 629-639, Jun. 2007, doi: 10.1243/09544119JEIM55.

5. "Indego Exoskeleton for Personal Use." http://www.indego.com/indego/us/en/indego\%2Dpersonal (accessed Sep. 29, 2021).

6. "ReWalk ${ }^{\mathrm{TM}}$ Personal 6.0 Exoskeleton For Spinal Cord Injury." https://rewalk.com/rewalk-personal-3/ (accessed Sep. 29, 2021).

7. "REX Product Information," Rex Bionics. https://www.rexbionics.com/product-information/ (accessed Sep. 29, 2021).

8. D. Arrienda, "EksoHealth," Ekso Bionics. https://eksobionics.com/eksoworks/ (accessed Sep. 29, 2021).

9. "ABLE Exoskeleton," ABLE Human Motion. https://www.ablehumanmotion.com/able-exoskeleton/ (accessed Sep. 29, 2021).

10. "SuitX Phoenix." https://www.suitx.com/phoenix-medical-exoskeleton (accessed Sep. 29, 2021). 
11. "Wandercraft Atalante." https://www.wandercraft.eu/ (accessed Sep. 29, 2021).

12. L. van Silfhout, A. J. F. Hosman, H. van de Meent, R. H. M. A. Bartels, and M. J. R. Edwards, "Design recommendations for exoskeletons: Perspectives of individuals with spinal cord injury," J. Spinal Cord Med., vol. 0, no. 0, pp. 1-6, Jun. 2021, doi: 10.1080/10790268.2021.1926177.

13. D. H. Gagnon, M. Vermette, C. Duclos, M. Aubertin-Leheudre, S. Ahmed, and D. Kairy, "Satisfaction and perceptions of long-term manual wheelchair users with a spinal cord injury upon completion of a locomotor training program with an overground robotic exoskeleton," Disabil. Rehabil. Assist. Technol., vol. 14, no. 2, pp. 138-145, Feb. 2019, doi: 10.1080/17483107.2017.1413145.

14. A. Kozlowski, T. Bryce, and M. Dijkers, "Time and Effort Required by Persons with Spinal Cord Injury to Learn to Use a Powered Exoskeleton for Assisted Walking," Top. Spinal Cord Inj. Rehabil., vol. 21, no. 2, pp. 110-121, Apr. 2015, doi: 10.1310/sci2102-110.

15. M. Arazpour et al., "Evaluation of a novel powered gait orthosis for walking by a spinal cord injury patient," Prosthet. Orthot. Int., vol. 36, no. 2, pp. 239-246, Jun. 2012, doi:

10.1177/0309364611435690.

16. C. Hartigan et al., "Mobility Outcomes Following Five Training Sessions with a Powered Exoskeleton," Top. Spinal Cord Inj. Rehabil., vol. 21, no. 2, pp. 93-99, 2015, doi: 10.1310/sci2102-93.

17. A. S. Gorgey, R. Wade, R. Sumrell, L. Villadelgado, R. E. Khalil, and T. Lavis, "Exoskeleton Training May Improve Level of Physical Activity After Spinal Cord Injury: A Case Series," Top. Spinal Cord Inj. Rehabil., vol. 23, no. 3, pp. 245-255, 2017, doi: 10.1310/sci16-00025.

18. A. D. Karelis, L. P. Carvalho, M. J. Castillo, D. H. Gagnon, and M. Aubertin-Leheudre, "Effect on body composition and bone mineral density of walking with a robotic exoskeleton in adults with chronic spinal cord injury," J. Rehabil. Med., vol. 49, no. 1, pp. 84-87, Jan. 2017, doi: 10.2340/165019772173.

19. C. M. Cirnigliaro et al., "Decreased total and central adiposity after 100 exoskeletal-assisted walking sessions in persons with chronic spinal cord injury," J. Clin. Densitom., vol. 21, no. 4, p. 601, Oct. 2018, doi: 10.1016/j.jocd.2018.05.013.

20. A. Esquenazi, M. Talaty, A. Packel, and M. Saulino, "The ReWalk Powered Exoskeleton to Restore Ambulatory Function to Individuals with Thoracic-Level Motor-Complete Spinal Cord Injury," Am. J. Phys. Med. Rehabil., vol. 91, no. 11, pp. 911-921, Nov. 2012, doi: 10.1097/PHM.0b013e318269d9a3.

21. A. W. Heinemann et al., "Experience of Robotic Exoskeleton Use at Four Spinal Cord Injury Model Systems Centers," J. Neurol. Phys. Ther., vol. 42, no. 4, pp. 256-267, Oct. 2018, doi: 10.1097/NPT.0000000000000235.

22. M. Juszczak, E. Gallo, and T. Bushnik, "Examining the Effects of a Powered Exoskeleton on Quality of Life and Secondary Impairments in People Living With Spinal Cord Injury," Top. Spinal Cord Inj. Rehabil., vol. 24, no. 4, pp. 336-342, 2018, doi: 10.1310/sci17-00055.

23. A. Palimaru, W. E. Cunningham, M. Dillistone, A. Vargas-Bustamante, H. Liu, and R. D. Hays, "A comparison of perceptions of quality of life among adults with spinal cord injury in the United States 
versus the United Kingdom," Qual. Life Res., vol. 26, no. 11, pp. 3143-3155, Nov. 2017, doi: 10.1007/s11136-017-1646-x.

24. N. B. Jain, M. Sullivan, L. E. Kazis, C. G. Tun, and E. Garshick, "Factors Associated with HealthRelated Quality of Life in Chronic Spinal Cord Injury," Am. J. Phys. Med. Rehabil. Assoc. Acad. Physiatr., vol. 86, no. 5, pp. 387-396, May 2007, doi: 10.1097/PHM.0b013e31804a7d00.

25. L. E. Miller, A. K. Zimmermann, and W. G. Herbert, "Clinical effectiveness and safety of powered exoskeleton-assisted walking in patients with spinal cord injury: systematic review with metaanalysis," Med. Devices Evid. Res., vol. 9, pp. 455-466, Mar. 2016, doi: 10.2147/MDER.S103102.

26. H. Fritz, D. Patzer, and S. S. Galen, "Robotic exoskeletons for reengaging in everyday activities: promises, pitfalls, and opportunities," Disabil. Rehabil., vol. 41, no. 5, pp. 560-563, Mar. 2019, doi: 10.1080/09638288.2017.1398786.

27. A. Cahill, O. M. Ginley, C. Bertrand, and O. Lennon, "Gym-based exoskeleton walking: A preliminary exploration of non-ambulatory end-user perspectives," Disabil. Health J., vol. 11, no. 3, pp. 478-485, Jul. 2018, doi: 10.1016/j.dhjo.2018.01.004.

28. R. B. van Dijsseldonk, I. J. W. van Nes, A. C. H. Geurts, and N. L. W. Keijsers, “Exoskeleton home and community use in people with complete spinal cord injury," Sci. Rep., vol. 10, no. 1, p. 15600, Sep. 2020, doi: 10.1038/s41598-020-72397-6.

29. R. B. van Dijsseldonk, H. Rijken, I. J. W. van Nes, H. van de Meent, and N. L. W. Keijsers, "A Framework for Measuring the Progress in Exoskeleton Skills in People with Complete Spinal Cord Injury," Front. Neurosci., vol. 11, p. 699, 2017, doi: 10.3389/fnins.2017.00699.

30. C. Kandilakis and E. Sasso-Lance, "Exoskeletons for Personal Use After Spinal Cord Injury," Arch. Phys. Med. Rehabil., vol. 102, no. 2, pp. 331-337, Feb. 2021, doi: 10.1016/j.apmr.2019.05.028.

31. Y. He, D. Eguren, T. P. Luu, and J. L. Contreras-Vidal, "Risk management and regulations for lower limb medical exoskeletons: a review," Med. Devices Auckl. NZ, vol. 10, pp. 89-107, 2017, doi: 10.2147/MDER.S107134.

32. E. Guanziroli, M. Cazzaniga, L. Colombo, S. Basilico, G. Legnani, and F. Molteni, "Assistive powered exoskeleton for complete spinal cord injury: correlations between walking ability and exoskeleton control," Eur. J. Phys. Rehabil. Med., vol. 55, no. 2, pp. 209-216, Apr. 2019, doi: 10.23736/S19739087.18.05308-X.

33. R. Riener, "The Cybathlon promotes the development of assistive technology for people with physical disabilities," J. NeuroEngineering Rehabil., vol. 13, no. 1, p. 49, May 2016, doi: 10.1186/s12984-0160157-2.

34. P. Wolf and R. Riener, "Cybathlon: How to promote the development of assistive technologies," Sci. Robot., vol. 3, no. 17, p. eaat7174, Apr. 2018, doi: 10.1126/scirobotics.aat7174.

35. K. Baur, F. L. Haufe, R. Sigrist, K. Dorfschmid, and R. Riener, "The CYBATHLON - Bionic Olympics to Benchmark Assistive Technologies," in Inclusive Robotics for a Better Society, Cham, 2020, pp. 175179. doi: 10.1007/978-3-030-24074-5_29. 
36. D. Novak et al., "Benchmarking Brain-Computer Interfaces Outside the Laboratory: The Cybathlon 2016," Front. Neurosci., vol. 11, p. 756, 2018, doi: 10.3389/fnins.2017.00756.

37. "Powered Exoskeleton Race." https://www.cybathlon.ethz.ch/en/event/disciplines/exo (accessed Sep. 29, 2021).

38. S. O. Schrade et al., "Development of VariLeg, an exoskeleton with variable stiffness actuation: first results and user evaluation from the CYBATHLON 2016," J. NeuroEngineering Rehabil., vol. 15, no. 1, p. 18, Mar. 2018, doi: 10.1186/s12984-018-0360-4.

39. P. Neuhaus, "IHMC's experience competing in the Cybathlon compared to the DARPA robotics challenge," J. NeuroEngineering Rehabil., vol. 14, no. 1, p. 112, Nov. 2017, doi: 10.1186/s12984-0170324-0.

40. J. Choi, B. Na, P.-G. Jung, D. Rha, and K. Kong, "WalkON Suit: A Medalist in the Powered Exoskeleton Race of Cybathlon 2016," IEEE Robot. Autom. Mag., vol. 24, no. 4, pp. 75-86, Dec. 2017, doi: 10.1109/MRA.2017.2752285.

41. T. Vouga, R. Baud, J. Fasola, M. Bouri, and H. Bleuler, "TWIICE - A lightweight lower-limb exoskeleton for complete paraplegics," IEEE Int. Conf. Rehabil. Robot., pp. 1639-1645, 2017, doi: 10.1109/ICORR.2017.8009483.

42. “ALULITE," Apr. 10, 2013. https://www.fischerconnectors.com/uk/en/products/alulite (accessed Oct. 01, 2021).

43. "Votre partenaire en mécatronique - Sonceboz." https://www.sonceboz.com/fr (accessed Oct. 01, 2021).

44. A. Yang, P. Asselin, S. Knezevic, S. Kornfeld, and A. Spungen, "Assessment of In-Hospital Walking Velocity and Level of Assistance in a Powered Exoskeleton in Persons with Spinal Cord Injury," Top. Spinal Cord Inj. Rehabil., vol. 21, no. 2, pp. 100-109, Apr. 2015, doi: 10.1310/sci2102-100.

45. C. Tefertiller et al., "Initial Outcomes from a Multicenter Study Utilizing the Indego Powered Exoskeleton in Spinal Cord Injury," Top. Spinal Cord Inj. Rehabil., vol. 24, no. 1, pp. 78-85, 2018, doi: 10.1310/sci17-00014.

\section{Figures}




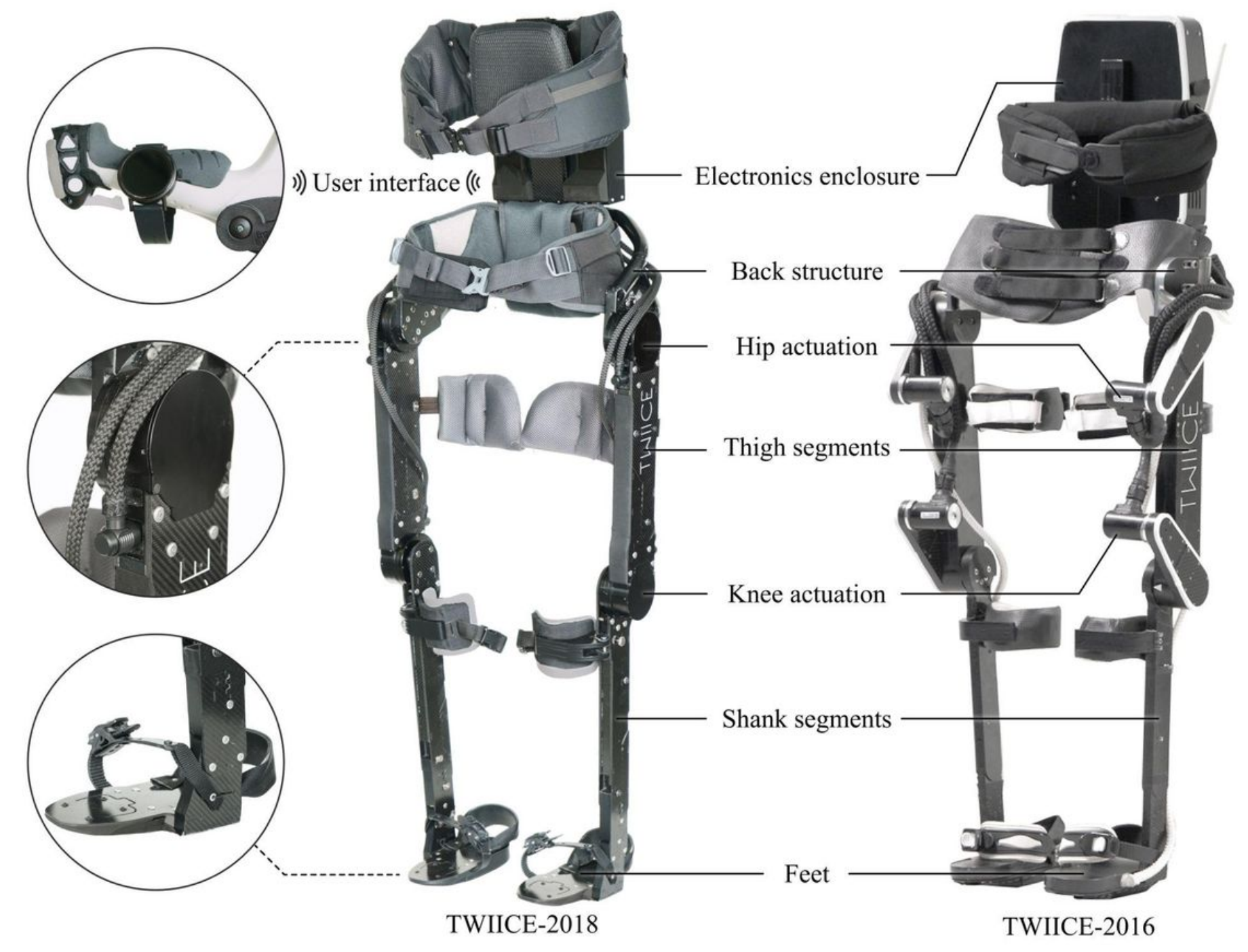

Figure 1

TWIICE one 2018 overview and a direct comparison with the 2016 version. The inlays, from top to bottom, show the user interface comprising a set of buttons as well as a trigger on the right crutch handle and a smartwatch as visual feedback, a zoom on the custom motors, and a zoom on the foot design. 

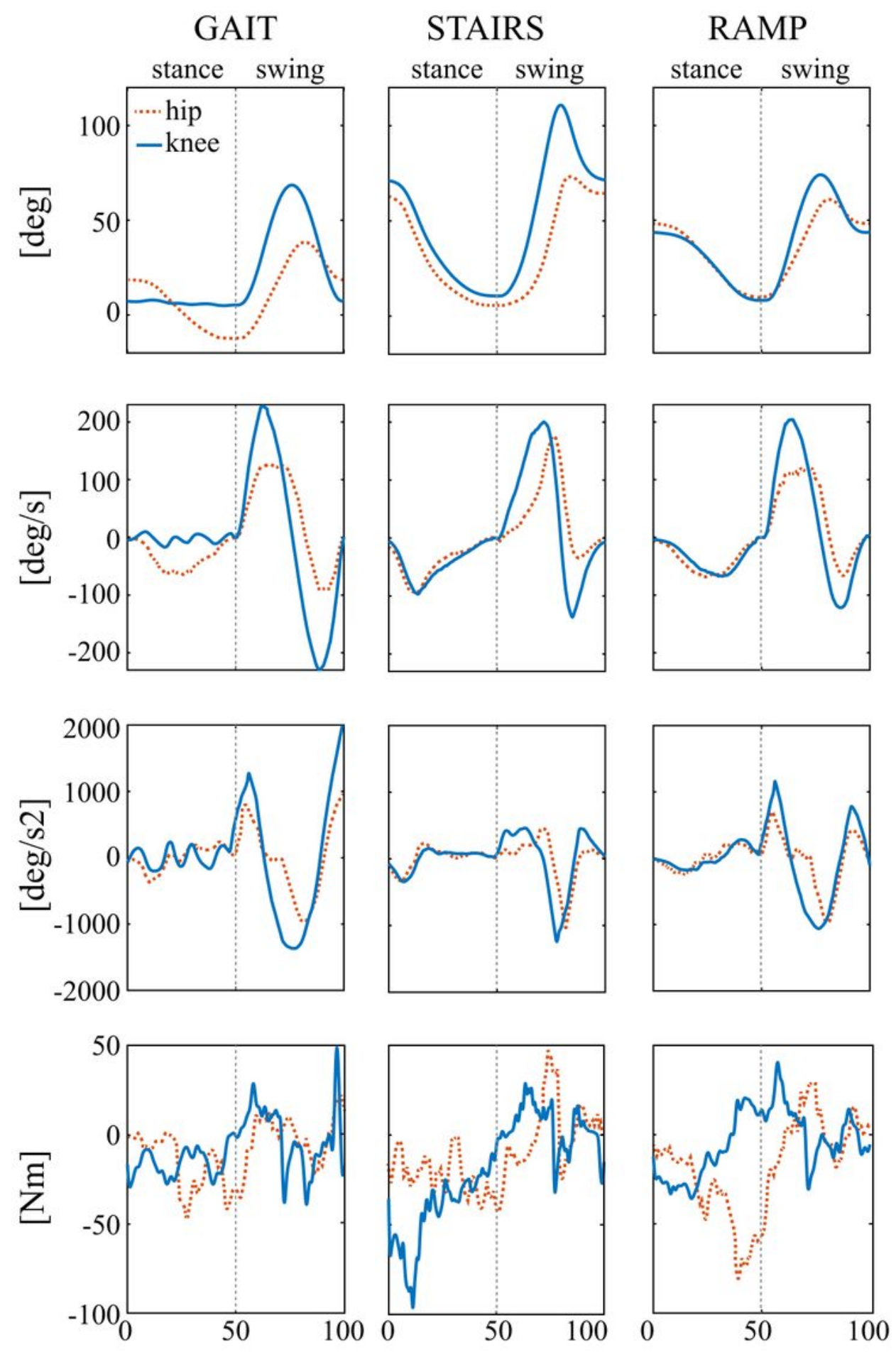

Percent of stride [\%]

\section{Figure 2}

Joint kinematics of the hip (dashed line) and the knee (solid line), their angular velocities and accelerations, and their torque for normal gait, stairs and ramp ascent represented as a function of stride. The vertical line represents a pause in the gait cycle between the stance and swing phases, corresponding to the time needed by the pilot to move the crutches forward. 
a)

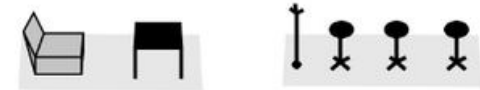

Sit \& Stand $\square$ Slalom

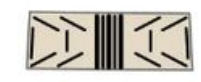

$\triangle$ Rough Terrain

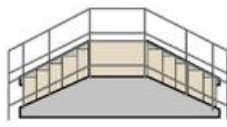

$\times$ Stairs

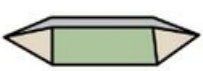

* Tilted Path

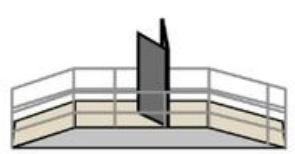

$\circ$ Ramp \& Door b)

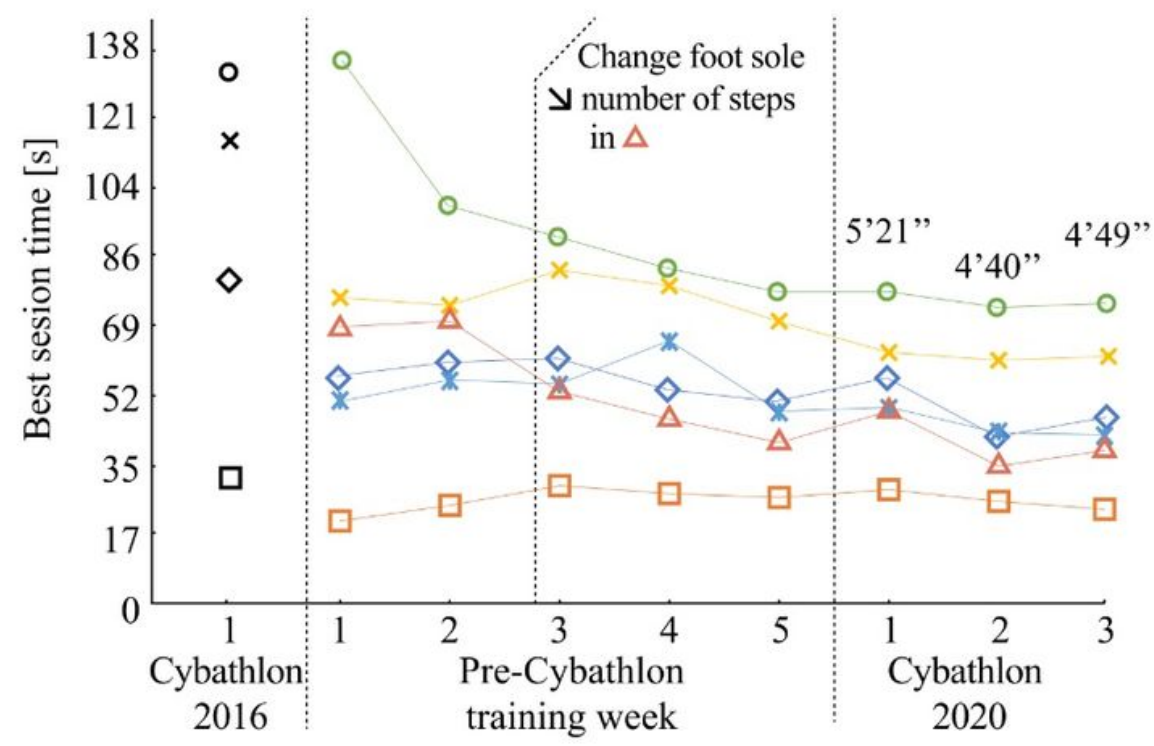

c)

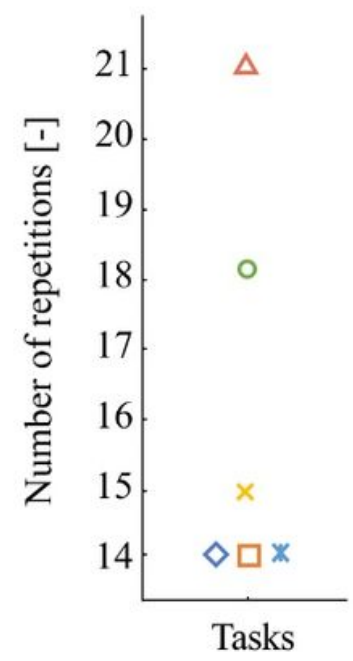

\section{Figure 3}

CYBATHLON obstacles training. a) The six CYBATHLON 2020 obstacles, matching the race order from left to right, are shown on the top of the figure. B) Best times per obstacle are shown. Although the obstacles of CYBATHLON 2016 were slightly different, their timing is given as a baseline. The whole race times are given for the 2020 competition. c) Number of training repetitions per obstacle during the preCYBATHLON week is shown 